\title{
Modeling, with a unified level-set representation, of the expansion of a hollow in the ground under different physical phenomena
}

\author{
Régis Cottereau · Pedro Díez · Antonio Huerta
}

Received: date / Accepted: date

\begin{abstract}
This paper builds on the flexibility of the level-set representation to model in a unified manner the expansion of a hollow in the ground under different physical phenomena. In particular, the dissolving action of a flow of water in a saturated soil, and that of a jet of particles of water in a non-saturated one, are represented in a common framework. In that manner, the complex geometrical evolutions of the hollow can be followed without the need for remeshing and this approach allows for a smooth transition between saturated and non-saturated models of the soil. Implementation and numerical difficulties are discussed and two applications of industrial interest are considered. The first one describes the modeling of the piping phenomenon, and the second one the evolution of an excavation created by a leaking duct.
\end{abstract}

Keywords Level-set method P Porous media · Piping · Internal erosion

\section{INTRODUCTION}

The requirement to capture the position and evolution of an interface is central to many areas of engineering and science,

The support of Gas Natural Distribución SDG S.A. and Ministerio de Educación y Ciencia de España, through grants DPI2007-62395 and BIA2007-66965, is gratefully acknowledged.

\section{Régis Cottereau}

Int. Center for Numerical Methods in Engineering (CIMNE)

E. T. S. d'Enginyers de Camins, Canals i Ports de Barcelona

Universitat Politècnica de Catalunya, Barcelona, Spain

\& Laboratoire MSSMat, CNRS UMR 8579

École Centrale Paris, Châtenay-Malabry, France

E-mail: regis.cottereau@ecp.fr

Pedro Díez · Antonio Huerta

Laboratori de Càlcul Numèric

Departament de Matemàtiques Aplicades III

Universitat Politècnica de Catalunya, Barcelona, Spain including metal forging, oceanography, imaging, flame modeling, melting of materials, and more generally, the modeling of heterogeneous or multi-phases materials. Along the years, several methods have been devised for such problems [1]. Among the most used ones are front tracking methods, in which the mesh is refined or deformed to follow the displacement of the interface [2], and the marker-in-cell method $[3,4]$, in which a large set of markers follows the material in a lagrangian way, hence describing the position of each phase. However, both methods induce high computational costs. Indeed, to keep the numerical accuracy to an appropriate level, it is necessary, in the former class of methods, to re-mesh the domain when the deformations increase. Likewise, the number of markers necessary to follow appropriately an interface is shown to be prohibitive for computational implementation [5].

Level-set functions $[6,7]$ provide a very efficient and elegant alternative to these techniques. In the simplest setting, they allow to discriminate between two areas of a domain, with no explicit parameterization of the actual interface. Level-sets are functions defined on the entire domain, whose sign indicates the belonging to one or the other of the two areas. Usually, their absolute value represents the smallest distance to the interface, which is hence indicated by the cancellation of the level-set. Conceptually, they are constructed in a space of higher dimension than the interface they intend to represent, with an improved mathematical behavior that allows for an easier manipulation.

Since the first use of level-set functions in the description of dynamical two-phase fluid systems [8,9], their power has been acknowledged for the parameterization of complex evolving phases. In particular, their ability to deal with changes in topology without any remeshing has been recognized $[10,11]$. They have been used in several fields of geophysics and geomechanics, including modeling of twophase flows and permeability estimation in reservoir simu- 
lations [11,12], tectonic plates subduction [13,14], seismic waves travel time computation [15], and, generally, for inverse problems and optimal design [16-18].

Apart from this potential in considering complex geometries, we want here to advocate the capabilities of the level set representation in integrating different physical models in a common framework. To our knowledge, this aspect has not been emphasized before. In particular, we consider a leaking duct, generating a hollow by dissolution and tearing of the soil. Depending on the height of the groundwater table, two very different physical phenomena occur. When the groundwater table is low, the hollow is full of air, and its expansion is mainly due to the impact of the jet of water coming out of the leak. When the groundwater table is high, the hollow is full of water, and the main phenomenon controlling the evolution of the excavation is the dissolution and transport of soil particles with the water flow. In this paper, both these phenomena will be considered in the same framework of the Hamilton-Jacobi equation. There, the choice of the model of erosion (jet or flow) is entirely contained in that of the velocity of advancement of the interface. Conceptually and practically, this change of focus with respect to the classical approach allows to shift very easily from one model of the advancement of the interface to the other. In real life (e.g., for the rise and fall of the water table in the soil), this may be a very common situation.

Note that this change of focus means that the questions of whether the models of normal velocity are actually useful for real-life applications, or whether the numerical schemes used to compute them are accurate, are of secondary importance for this paper. We will concentrate on the integration of both models in a common framework, and on the numerical accuracy of the level set representation itself. The latter will be described on an example representing the piping phenomenon, with only one model involved. Apart from its interest in showing the numerical accuracy of the level set representation, this example is also, to the knowledge of the authors, the first application of level sets to this problem, for which it seems appealing. Indeed, piping is one the main causes of failure of dams and embankments, while its modeling is still based on semi-analytical [19] or experimental [20,21] approaches. In particular, the semi-analytical approaches are heavily dependent on symmetry hypotheses that are probably not very relevant in practice. Using a levelset parameterization for the interface between the soil and the hollow allows to follow any geometry of the piping hole.

The paper is constructed as follows. In a first part (section 2), we recall the main definitions and results for level set descriptions, and introduce two examples of models for the normal velocity of the interface (sections 2.3 and 2.4). These two models are the ones that are used in the examples of the last section. We then describe some numerical issues related to the use of level-set descriptions (section 3), in particu-
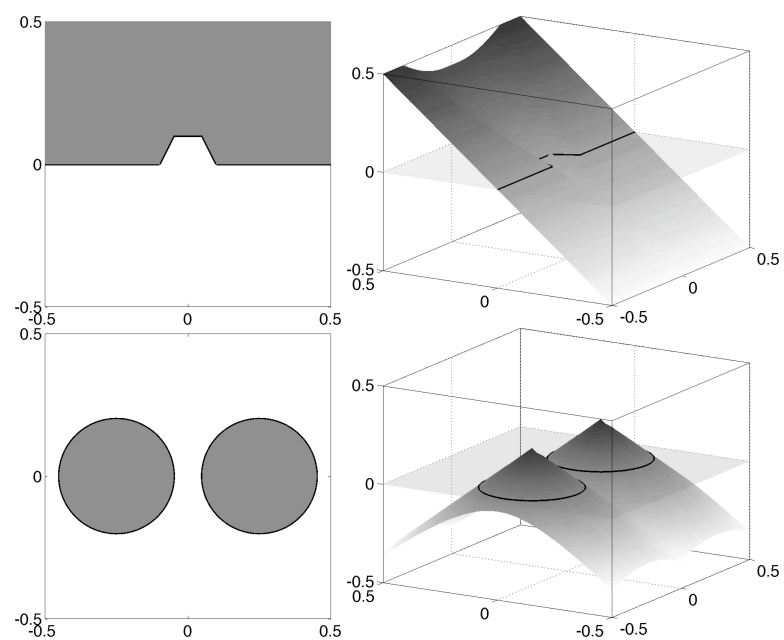

Fig. 1 Two examples of 2D two-phases system (left) with the corresponding level-set functions (right)

lar concerning the size of the mesh and re-distancing of the level-set function. Finally, we present the two applications of interest: a level-set description of the piping phenomenon (section 4.2), and the expansion of a hollow in the ground under two different physical phenomena (section 4.3).

\section{THE LEVEL-SET DESCRIPTION}

The goal of this section is to provide a general description of the level-set approach for the representation of a multi-phase medium. The original ideas were presented in [8]. Although we only discuss here the case where there are two phases, the ideas can be extended to multi-phases problems [22].

\subsection{Basic principles}

Let us consider a domain $\Omega$ separated into exactly two subdomains $\Omega_{1}$ and $\Omega_{2}$. The interface between these two subdomains is denoted $\Gamma$ and evolves in time with a normal velocity $\mathbf{v}_{n}(\mathbf{x}, t)$. Note that the definition of this velocity field implies the choice of an "interior" and "exterior". Two examples of such situations, with closed and open interfaces, are shown in the left column of figure 1.

The main idea of the level-set approach is to consider a function, defined on the entire domain of interest, with values that depend on the position in one phase or the other. The most common approach consists in defining the levelset function $\Phi$ as the distance function to the interface, with the sign indicating the subdomain. In mathematical terms, it 
is defined by

$$
\begin{cases}|\Phi(\mathbf{x})|=\ell(\mathbf{x}) & \\ \Phi(\mathbf{x})>0 & \mathbf{x} \in \Omega_{1} \\ \Phi(\mathbf{x})<0 & \mathbf{x} \in \Omega_{2} \\ \Phi(\mathbf{x})=0 & \mathbf{x} \in \Gamma\end{cases}
$$

where $\ell(\mathbf{x})$ indicates the distance from point $x$ to the closest point of curve $\Phi=0$. Hence, the level-set function is defined in a space with a higher dimension than the interface it attempts to parameterize. However, the additional dimension allows for an increased smoothness of the function, which can hence be more easily manipulated. Further, the level-set description of the interface fits properly in a Finite Element (FE) context because the function $\Phi$ may be interpolated using the same mesh. This allows describing arbitrary interfaces, not restricted to contain the mesh nodes, with an accuracy and regularity up to the resolution of the FE discretization. The level-set functions corresponding to two examples of interfaces are shown in figure 1. In these two examples, the interfaces are lines (1D) in a 2D space, and the level-set functions are surfaces (2D) in a 3D space.

\subsection{The Hamilton-Jacobi equation}

As the phases of the model evolve in time, so does the interface and its level-set description. The evolution of the levelset function $\Phi$ is determined by the normal velocity of the interface at every point, $v_{n}(\mathbf{x}, t)$. This front velocity is considered to be positive if the interface advances towards the positive values of $\Phi$, that is in the direction of the unit normal vector $\mathbf{n}=\nabla \Phi /|\nabla \Phi|$. The transport equation for $\Phi$ is the Hamilton-Jacobi equation, and its derivation, given the front velocity $v_{n}(\mathbf{x}, t)$, is sketched below.

Let us consider a point $\mathbf{x}(t)$ following the interface in its movement. Thus, at every time $t$,

$\Phi(\mathbf{x}(t), t)=0$

The time derivative of this equation yields

$\frac{\partial \Phi}{\partial t}+\nabla \Phi \cdot \frac{\partial \mathbf{x}}{\partial t}=0$

Recalling that the front velocity is precisely $v_{n}(\mathbf{x}, t)=\mathbf{n}$. $\partial \mathbf{x} / \partial t$, with $\mathbf{n}=\nabla \Phi /|\nabla \Phi|$, the second term in the lefthand-side of the previous equation is replaced by $v_{n}(\mathbf{x}, t)|\nabla \Phi|$. This results in the Hamilton-Jacobi equation:

$$
\frac{\partial \Phi}{\partial t}+v_{n}(\mathbf{x}, t)|\nabla \Phi|=0
$$

Note that, to this point, no hypothesis has been made on the form of the normal velocity field $v_{n}$. This equation is therefore compatible with different physical models of the evolution of the interface. Conversely, the only necessary modification for passing from one model to another is to change the form of the normal velocity. This is the property that is stressed in this paper, and that is, to the belief of the authors, a yet under-rated advantage of the level-set representation. It allows for a very easy shifting, possibly back and forth, between one model and another.

Thereafter, we present two models of erosion that can be used within this general framework. By model of erosion, what is meant is the description of the normal velocity of the interface in different physical situations. We namely consider two of them: the erosion by a jet of water of an airfilled cavity within an earth matrix, and the erosion by a flow of water of a water-filled cavity. Note that the forms of these two models are very different and that they are solved by very different schemes. This is typically the situation when it appears interesting to use a unified framework using the level-set parameterization. As already stated above, and because the choice of these models is not central to the focus of this paper, we will not elaborate on their validation by experimental evidence, or on the numerical accuracy of the schemes used to compute the values of the normal velocities.

\subsection{Erosion due to a jet}

The first physical situation we consider is that of the erosion by a jet of water of an air-filled cavity within the earth. The jet of water is modeled as a set of ejected particles (small volumes of water, drops), launched at different times and from different points of a source. The volumes of the different particles are denoted by $V_{p}, p=1,2, \ldots$, such that the particles move through space as point masses $m_{p}=\rho_{w} V_{p}$, where $\rho_{w}$ is the density of water. The source corresponds geometrically to a small part of the boundary. Each particle undergoes a free flight, only submitted to gravity, starting at time $t_{p}^{0}$ from a point $\mathbf{x}_{p}^{0}$ of the source and with an initial velocity $\mathbf{v}_{p}^{0}$. Thus, the position $\mathbf{x}_{p}(t)$ and velocity $\mathbf{v}_{p}(t)$ of a particle $p$ at time $t$ are given by

$\left\{\begin{array}{l}\mathbf{x}_{p}(t)=\mathbf{x}_{p}^{0}+\mathbf{v}_{p}^{0}\left(t-t_{p}^{0}\right)+\mathbf{g} \frac{1}{2}\left(t-t_{p}^{0}\right)^{2}, \\ \mathbf{v}_{p}(t)=\mathbf{v}_{p}^{0}+\mathbf{g}\left(t-t_{p}^{0}\right)\end{array}\right.$,

where $\mathbf{g}=-g \mathbf{i}_{z}$ is the acceleration of gravity, with $g=9.81$ $\mathrm{m} / \mathrm{s}^{2}$, and $\mathbf{i}_{z}$ points upwards.

For a given time interval $[t-\Delta t / 2, t+\Delta t / 2]$, the flow is modeled using a given number of particles $N$, to each of which is assigned the same mass and velocity (norm), so as to fit the macroscopic jet values, in particular the flow, $Q$ in $\mathrm{m}^{3} / \mathrm{s}$, and the velocity, in $\mathrm{m} / \mathrm{s}$. Both the location $\mathbf{x}_{p}^{0}$ and the direction (angle) of the initial velocity $\mathbf{v}_{p}^{0}$ are selected randomly using a proper assumption in their probability distribution (for instance, uniform density for the location, Gaussian distribution for the direction). Tuning the parameters 
of these probability distributions allows modeling wider or narrower jets, with different dispersions. The velocity of the particles is assumed to be large, in the sense that the flying time of the particles is much lower than the time interval $\Delta t$. Under this assumption, we concentrate the launching of all the particles at the mid time of the interval, namely $t_{p}^{0}=t$, for $p=1,2, \ldots, N$.

Given the level-set parametrization $\Phi(\mathbf{x})=0$ of the interface between the earth and the air, we then calculate the point and velocity of impact of each particle with the interface as the first time $t_{p}^{i}$ (after $t_{p}^{0}$ ) when the particle verifies $\Phi\left(\mathbf{x}_{p}\left(t_{p}^{i}\right)\right)=0$, that is

$$
\left\{\begin{array}{l}
t_{p}^{i}=\min _{t>t_{p}^{0}}\left\{\arg \Phi\left(\mathbf{x}_{p}(t)\right)=0\right\} \\
\mathbf{x}_{p}^{i}=\mathbf{x}_{p}\left(t_{p}^{i}\right)=\mathbf{x}_{p}^{0}+\mathbf{v}_{p}^{0}\left(t_{p}^{i}-t_{p}^{0}\right)+\mathbf{g} \frac{\left(t_{p}^{i}-t_{p}^{0}\right)^{2}}{2} \\
\mathbf{v}_{p}^{i}=\mathbf{v}_{p}\left(t_{p}^{i}\right)=\mathbf{v}_{p}^{0}+\mathbf{g}\left(t_{p}^{i}-t_{p}^{0}\right)
\end{array} .\right.
$$

When using a FE approach for the representation of the levelset function, as described in section 3 , finding $\mathbf{x}_{p}^{i}$ and $\mathbf{v}_{p}^{i}$ reduces to finding the intersection of the parabolic trajectory of the particle $p$ with the polygonal interface described by the FE approximation to $\Phi(\mathbf{x})$ (this polygonal interface corresponds to linear elements, and is replaced by a piecewise quadratic curve for elements of degree two). In the general case, this intersection can be located very easily by simply sampling the level set function along the $1 \mathrm{D}$, curved, trajectories of the particles, and detecting the first change of sign.

We then choose to model the erosion by taking the normal velocity of the interface as proportional to an averaged normal impacting momentum. Thus, in order to assess this normal velocity, at a given point $\mathbf{x}$ of the interface, and at time $t, \mathbf{v}_{n}(\mathbf{x}, t)$, we consider the particles impacting during a short time interval $\Delta t$ a small surface (segment in 2D) $d S_{\mathbf{x}}$ with normal $\mathbf{n}$, centered around $\mathbf{x}$. The average is taken as:

$\mathbf{v}_{n}(\mathbf{x}, t)=\frac{\mathscr{V}_{0}}{d S \Delta t} \sum_{\mathscr{P}} \mathbf{n} \cdot \mathbf{v}_{p}^{i} m_{p}$

where $\mathscr{P}$ is the set of particles that hit the interface on surface $d S_{\mathbf{x}}$ and in the time span $[t-\Delta t / 2, t+\Delta t / 2]$, that is $\mathscr{P}=\left\{p \mid \mathbf{x}_{p}^{i} \in d S_{\mathbf{x}}, t_{p}^{i} \in[t-\Delta t / 2, t+\Delta t / 2]\right\}, \mathscr{V}_{0}$ is a parameter that depends on the cohesion of the material, and $d S=\left|d S_{\mathbf{x}}\right|$ is the measure of the surface $d S_{\mathbf{x}}$. If one additionally supposes that all the particles have the same mass $m=\rho_{w} Q(t) \Delta t / N$, where $Q(t)$ is the flow of water coming out of the jet at time $t$, this equation simplifies to

$\mathbf{v}_{n}(\mathbf{x}, t)=\frac{\rho_{w} \mathscr{V}_{0} Q(t)}{N d S} \sum_{\mathscr{P}} \mathbf{n} \cdot \mathbf{v}_{p}^{i}$

This formulation is well adapted to a Monte Carlo resolution because the number $N$ of particles of water in which the jet has been separated appears explicitely.

\subsection{Erosion due to a flow}

For this second model problem, we consider water flowing through a water-filled cavity and a soil. The flow of water is modeled using Darcy's law [23], which, together with a mass balance, specifies the evolution of the hydraulic head $h(\mathbf{x}, t)=z+P(\mathbf{x}, t) / \rho_{w} g$, with $z$ the altitude and $P(\mathbf{x}, t)$ the hydraulic pressure,

$\nabla \cdot(K(\mathbf{x}, t) \nabla h(\mathbf{x}, t))=\mathbf{0}$,

The permeability $K(\mathbf{x})$ describes how easily the water flows through the medium. Note that we consider here a quasistatic problem, so that we neglected a possible influence of $\partial h / \partial t$. However, it would be possible to include dynamic effects through the inclusion of a time derivative term [24].

The two domains of our problem, namely the soil and the cavity, are modeled using two different permeability values

$\left\{\begin{array}{l}K\left(\mathbf{x} \in \Omega_{s}, t\right)=K\left(\mathbf{x} \in \Omega_{s}\right)=K_{s} \\ K\left(\mathbf{x} \in \Omega_{c}, t\right)=K\left(\mathbf{x} \in \Omega_{c}\right)=1000 K_{s}\end{array}\right.$

where $\Omega_{s}$ and $\Omega_{c}$ are respectively the domain occupied by the soil and the water-filled cavity, such that $\Omega_{s} \cup \Omega_{c}=\Omega$. Note that we suppose that the permeability coefficients do not explicitly depend on the time variable. However, as the definitions of the domains $\Omega_{s}$ and $\Omega_{c}$ does, then the value of the permeability fields in a given point in space will change with time.

In practice, this equation is solved using a FE method. Other methods, in particular the eXtended FE (XFE) method combined with the level-set representation of the interface for the discretization in space, could have been used to increase the accuracy of the approximation (see section 3.3 for more details).

We now describe two models of flow erosion, designed respectively for flows parallel and normal to the surface that is being eroded. In the literature, the former type of model appears much more often, due to its importance in the creation of gullies and rills, which is an important issue for agriculture in particular (see [25] for a general review). Models dealing with the erosion of a surface under a normal flow are more scarce, and related to problems of the oil industry [26, 27].

Starting with models for perpendicular flows, the most widely used describes the rate of erosion per surface area $\dot{\varepsilon}$ (sometimes denoted $D_{c}$ ) as proportional to the hydraulic shear stress $\tau$ [25]:

$\dot{\varepsilon}=K_{c}\left(|\tau|-\tau_{c r}\right)$,

where $K_{c}$ is the coefficient of erosion (with values ranging from $10^{-1}$ to $10^{-6} \mathrm{~s} . \mathrm{m}^{-1}$ [20]) and $\tau_{c r}$ is a critical shear stress (with values ranging from 6 to $160 \mathrm{~N} . \mathrm{m}^{-2}$ [21]), both material-dependent. Other models replace the shear stress 
by the stream power as the controlling parameter but experimental evidence seems to indicate that the shear force model is appropriate, both for laboratory and in situ cases [28]. More refined models try to include the influence of the sediments detached from the soil (see for example [29, 19]), or consider probabilistic models for the erosion factors [30], however it remains unclear whether the alternatives they provide are truly required with respect to the simpler model of Eq. (11). Following [20,25], an approximation can be used to derive the hydraulic shear stress as a function of the gradient of the hydraulic head,

$\tau=\rho_{w} g \ell_{0} \nabla h(\mathbf{x}, t)$

where $\ell_{0}=1 \mathrm{~m}$ in $2 \mathrm{D}$ problems. This finally leads to a normal velocity for the cavity-soil interface

$v_{n}(\mathbf{x}, t)=\frac{\dot{\varepsilon}}{\rho_{s}}=\frac{K_{c}}{\rho_{s}}\left(\rho_{w} g \ell_{0}|\nabla h(\mathbf{x}, t)|-\tau_{c r}\right)$,

where $\rho_{s}$ is the density of the soil material.

In the case of an erosion taking place along the axis of the flow, it is not reasonable to use the shear stress as a controlling parameter for erosion. Following [26], and simplifying the model described there because we are only interested in the erosion at the interface between the cavity and the soil, we get a linear relation between the rate of erosion per surface area and the norm of the Darcy flow $q_{n}=K_{s} \nabla h$, which leads to

$v_{n}(\mathbf{x}, t)=\frac{\dot{\varepsilon}}{\rho_{s}}=\lambda|\nabla h|$,

where $\lambda$ is a proportionality coefficient, that would have to be determined experimentally. If we neglect $\tau_{c r}$ in Eq. (13), then the two equations for the normal velocity have the same form (although derived for different directions), but with different proportionality coefficients a priori. As the real flow going through an interface is probably in between that of a normal incidence and grazing incidence, we will consider in all cases a model for the normal velocity as in Eq. (14), with the additional hypothesis that the proportionality coefficient is the same for all incidences. A more refined model would require the identification of the coefficient as a function of the angle of incidence.

\section{NUMERICAL ISSUES}

In this section, we discuss several numerical points that arise when dealing with level-set methods. In particular, the sections 3.1 and 3.2 describe the important issues of, respectively, the influence of the size of the mesh on the representation of the interface, and the requirement of regular renormalization of the distance function.

\subsection{Numerical description of the level-set}

To this point, the level-set function $\Phi(\mathbf{x})$ was defined as a general field. However, in practice, the level-set function is represented as a FE approximation over a mesh, and described by the nodal values of $\Phi$. This means that the position of the interface $\Phi=0$ is never explicitly stored. Rather, it is recovered, when necessary, by interpolation of the values of $\Phi\left(\mathbf{x}_{n}\right)$ at the nodes $\mathbf{x}_{n}$ of the mesh. When using linear finite elements, the interface is therefore approximated inside each element it crosses by a straight line. Hence, strong curvatures of the interface may be smoothed out if a coarse mesh is used.

Note that this representation is totally independent of any FE scheme that might be used for the computation of the normal velocity that appears in the Hamilton-Jacobi equation. In particular, when using the model for the erosion by a flow of water (section 2.4), it is possible to use two different meshes: one for the computation of the normal velocity, and one for representing the level-set function. In that case, it is usually interesting to use a mesh much more refined for the representation of the level-set than for the resolution of the flow problem required to compute the front velocity. Due to the explicit character of the method introduced in section 3.2, the additional cost of the level-set transport associated with the mesh refinement is not significant. Moreover, the refined mesh can easily be obtained from a coarse one because there is no requirement of shape for the elements. As they are not used for integration purposes, they can be as deformed as required by the needs of the representation of the curvature.

However, in practice, when a FE scheme is used for the computation of the normal velocity, the two meshes are often taken as the same. This is mainly dictated by the simplicity of the numerical implementation, and this skips an interpolation step that would be otherwise necessary to pass the information about the position of the interface from one mesh to the other. Note that, when using only one FE mesh, one should pay attention to the fact that the size of the elements of the mesh should be small enough with respect to the smoothness of both the interface and the FE solution that is being computed (the hydraulic head in the case of the erosion by a flow). Note, also, that this technique was not implemented in the examples of section 4, because the mesh used for the resolution of Darcy's equation was fine enough for the representation of the curvature of the interface.

3.2 Transporting the level set: solving the Hamilton-Jacobi equation

Once $v_{n}\left(\mathbf{x}_{j}, t\right)$ is obtained from the models discussed in sections 2.3 and 2.4, the interface has to be updated solving the Hamilton-Jacobi equation (4). Recall that this is a nonlinear 
hyperbolic advection problem. A summarized description of the general numerical approaches to tackle this problem using FE may be found in [31], and ad-hoc strategies for the particular case of Hamilton-Jacobi are discussed in detail in [6]. The latter are based on upwind schemes for regular grids. Here, we present a new technique keeping the upwind approach in a general FE context. It is usable with unstructured meshes and allows the use of adaptivity techniques.

First, the Hamilton-Jacobi equation (4) is discretized along the time dimension using a first-order forward Finite Difference method. We consider that the level-set function at one point $t_{\ell}$ of the time grid, $\Phi_{\ell}=\Phi\left(\mathbf{x}, t_{\ell}\right)$, is known, and we look for the value of $\Phi$ at the next point in the grid, $t_{\ell+1}$. Thus, the Hamilton-Jacobi equation is approximated at the time $t$ in the span $\left[t_{\ell}, t_{\ell+1}\right]$ as

$$
\frac{\Phi_{\ell+1}-\Phi_{\ell}}{\Delta t}+v_{n}(\mathbf{x}, t)|\nabla \Phi(\mathbf{x}, t)|=0,
$$

where $\Delta t=t_{\ell+1}-t_{\ell}$. Hence, selecting the sampling time $t$ equal to $t_{\ell}$ (forward Euler type explicit scheme), the semidiscretized Hamilton-Jacobi equation reads

$\Phi_{\ell+1}=\Phi_{\ell}-v_{n}\left(\mathbf{x}, t_{\ell}\right) \Delta t\left|\nabla \Phi_{\ell}\right|$,

and $\Phi_{\ell+1}$ can be solved for, given $v_{n}\left(\mathbf{x}, t_{\ell}\right)$ and $\Phi_{\ell}$.

As previously mentioned, the space discretization strategy is adapted to exploit the information contained in the FE description of $\Phi$. We are not using however a standard FE approach, in the sense that the evolution of the nodal values of $\Phi$ is computed independently, in a node-by-node basis. This results in an explicit low-cost algorithm. In this paper, we use linear FE interpolation functions, so that $\left|\nabla \Phi_{\ell}\right|$ is not defined at the nodes of the mesh, but rather on each element of that mesh. Therefore, the values at the nodes $|\nabla \Phi|_{j, \ell}=|\nabla \Phi|\left(\mathbf{x}_{j}, t_{\ell}\right)$ are approximated by least-square fitting over the elements surrounding the node $\mathbf{x}_{j}$. It is worth noting that the least squares fitting strategy is a very flexible tool. For instance, it allows introducing the upwind by simply increasing the weight of the elements located upstream from the point of interest. This is an attractive feature of the methodology because as pointed out by many authors [6], upwinding is required to stabilize the transport schemes in the presence of sharp fronts. In the application examples we include in the next section, however, the solutions are pretty stable and upwinding did not introduce any significant improvement. The discretized form of the Hamilton-Jacobi equation is then

$\Phi_{j, \ell+1}=\Phi_{j, \ell}-v_{n}\left(\mathbf{x}_{j}, t_{\ell}\right) \Delta t|\nabla \Phi|_{j, \ell}$,

Note that, following the remark of the previous section, it might be very interesting here to use a mesh for the representation of the level-set function independent from the one used to compute the normal velocity field. This new mesh could be structured and therefore allow for the use of FD schemes for the discretization along the space dimension. This may provide for substantial savings of computational power.

The derivation of the Hamilton-Jacobi equation stems from two basic hypotheses: (1) the space points for which the equation is defined are along the interface $\Phi=0$, and (2) the level-set function is a distance function. This means that the Hamilton-Jacobi equation should not be solved on the entire domain but rather only close to the interface. Indeed, by solving it on the entire domain, there is no certainty that the level-set obtained would remain a distance function to the interface. This is particularly critical when the controlling parameters on the two sides of the interface are very different (in this paper, the ratio of permeability coefficients is 1000). After having moved the interface (by modifying the value at the nodes close to that interface), the value of the level-set function at all other nodes should therefore be obtained through the actual computation of the distance function. Note that this is a computationally involved step for which specific methods can be implemented [32,33]. Note also that some authors $[9,34]$ have devised iterative algorithms to solve the Hamilton-Jacobi equation on the entire domain, with the added objective of obtaining directly a distance function for the solution.

\subsection{FEM or XFEM}

Recently, level-set descriptions for interfaces have been used extensively in the context of XFE methods [13,22,35,36]. These methods build on the parameterization of the level-set to enrich the FE basis using functions that contain information on the interface. When the computation of the normal velocity appearing in the Hamilton-Jacobi equation (17) is performed using a FE method (for example in the case of the erosion by a flow), these approaches can be used straightforwardly. Indeed, as the level set function is already defined for the Hamilton-Jacobi equation, it may be re-used, with no additional cost, for the introduction of the XFE method. Intuitively, the enriched XFE solution would increase the accuracy of the numerical solution of the Darcy equation (9), and hence of the approximation of the normal velocity. However, one must be careful that we are interested here in the gradients of the hydraulic head, rather than in the hydraulic head itself, and the use of the XFE method does not necessarily induce better gradients. In the cases considered in section 4, the use of a XFE method did not seem to improve substantially the results, so that we only present results using the classical FE method. Some leads on the subject of accuracy of the XFE method can be found in [37,38]. In any case, this XFE method is used only for the computation of the normal velocity, which, as stated earlier, is secondary to the technique described in this paper. 


\section{APPLICATIONS}

In this section, we will present two applications: one that aims at showing the accuracy of the level-set representation, and the other one at illustrating the flexibility of the levelset method for incorporating different physical models in a common framework. The latter application provided the original motivation for this work. In the former application, modeling the piping phenomenon, level-set methods have never been applied before, to the knowledge of the authors.

\subsection{Methodology}

The general methodology used for both applications is the following:

1. Set the initial level-set function $\Phi\left(\mathbf{x}_{i}, t_{0}\right)$ for all nodes $\mathbf{x}_{i}$ of the mesh

2. At each time step $t_{n}=t_{0}+n \Delta t$

(a) Compute the value of the normal velocity $v_{n}\left(\mathbf{x}_{i}, t_{n}\right)$ for all nodes $\mathbf{x}_{i}$, using one of the two available models, that is Eq. (8) or Eq. (14).

(b) Solve the discretized Hamilton-Jacobi equation (17) and get $\Phi\left(\mathbf{x}_{i}, t_{n}\right)$ for all nodes $\mathbf{x}_{i}$

(c) Reshape the level-set function $\Phi\left(\mathbf{x}_{i}, t_{n}\right)$ as a distance function from $\Phi\left(\mathbf{x}, t_{n}\right)=0$ (see section 3.2)

\subsection{Modeling of the piping effect}

The level-set parameterization is applied to try to represent the piping phenomenon. This physical process takes place in dams, levees and dykes, and is responsible for a large number of their failures. The control of this phenomenon is becoming increasingly important, in particular in the context of global heating and the subsequent droughts and floods that accompany it. The piping denotes the expansion of a small canal within a block of soil, under the eroding action of the water flowing through it.

Several authors investigated this phenomenon, starting with the leading works of Bligh [39] and Lane [40], often concentrating on the construction of global indicators of the occurence of piping $[41,42]$. Recent papers try to propose refined analyses, by modeling more accurately the transport of the eroded sediments and the flow of water $[19,43,44]$. These works are encouraging as they provide a means to reproduce extremely well experimental data [20,21]. However, as they are semi-analytical, they are limited in the type of problems they can address. In particular, they require the section of the pipe to be circular and the flow to be axisymmetrical. The use of level-set methods would allow to generalize this type of approach to more complicated and realistic situations. Further, experimental means of detecting in

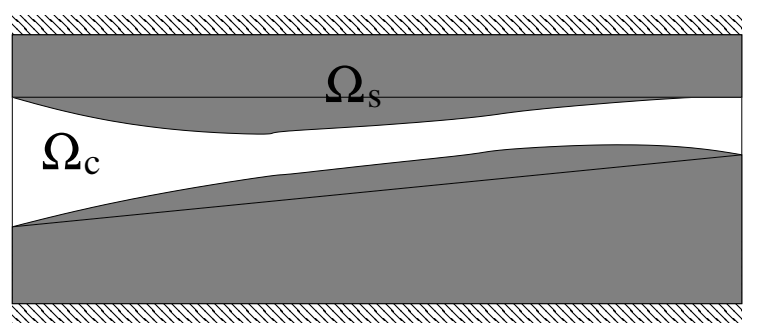

Fig. 2 Modeling of the piping phenomenon: a small channel $\Omega_{c}$ in a mass of soil $\Omega_{s}$ opens up under the eroding action of the water flowing from the right to the left.

situ the occurrence of piping are being developed. For example, in [45], the authors describe the use of an electrical probe that measures the conductivity of a mass of soil and can assess the presence of a water-filled cavity by solving an inverse problem. However, as the authors themselves state, the resolution of that inverse problem may be strongly influenced by the geometry of the sample. The level-set parameterization of the cavity may also help in that objective.

We model the piping phenomenon by describing the evolution of a pipe $\Omega_{c}$ in a mass of soil $\Omega_{s}$ (see figure 2)). The flow of water through both domains is modeled using Darcy's equation, and the expansion of the hole is provoked by that flow, as in section 2.4 (note that in this first application, there is only one model of erosion involved). The boundary conditions for that problems are

$\begin{cases}h(\mathbf{x}, t)=1, & \mathbf{x} \in \Gamma_{\text {right }} \\ h(\mathbf{x}, t)=0, & \mathbf{x} \in \Gamma_{\text {left }} \\ \nabla h(\mathbf{x}, t)=\mathbf{0}, & \mathbf{x} \in \Gamma_{\text {up }} \cup \Gamma_{\text {bottom }}\end{cases}$

so that the gradient of hydraulic head between the left and right sides will create a flow from the right side to the left side. The size of the domain is $2 \times 2 \mathrm{~m}^{2}$, and we consider two different initial channels: the first one with a constant radius $r_{0}=20 \mathrm{~cm}$ (figure $3(\mathrm{a})$ ) and the other one with a sine profile of amplitude $s=5 \mathrm{~cm}$ and wavelength $l=50 \mathrm{~cm}$ around a cylinder of constant radius $r_{0}$ (figure $3(\mathrm{~d})$ ). The erosion process takes place as described in section 2.4 , with $\lambda=10^{-2} \mathrm{~m} . \mathrm{s}^{-1}$ in Eq. (14).

We first present preliminary results, showing the interest of using the level-set parameterization in the modeling of the piping effect. In particular, the expansion of the pipe for the two initial profiles is described in figure 3 . The first line presents the case classically treated in the literature, and for which experiments are available [20,21]. Using these experiments, the parameters of the erosion models ( $\lambda$ in our case, but other erosion models could be used) can be identified. Once the parameters are identified, the model could then be used for more complicated initial profiles, such as that of the second line in figure 3. It is important to note that the extension to other geometries and fully 3D models is not a complicated task in the context of level-set methods. 


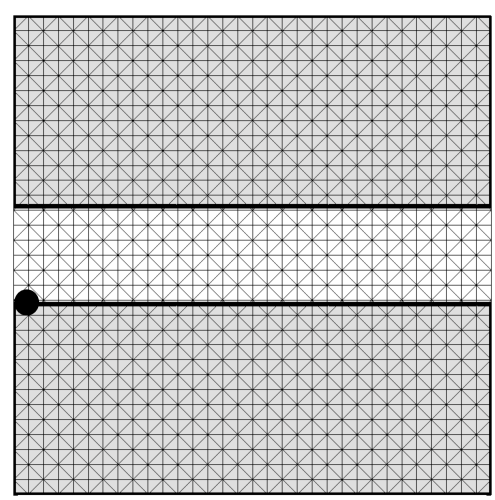

(a) $t=0 \mathrm{~s}$

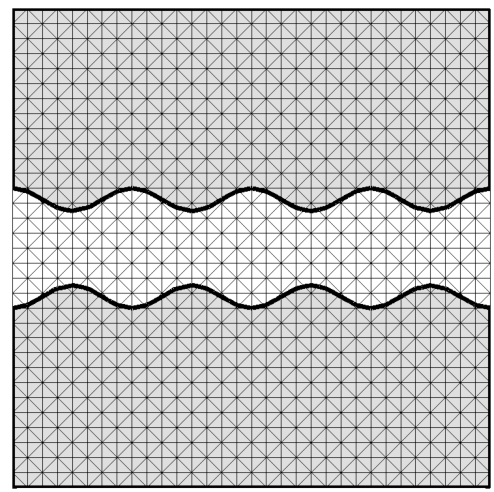

(d) $t=0 \mathrm{~s}$

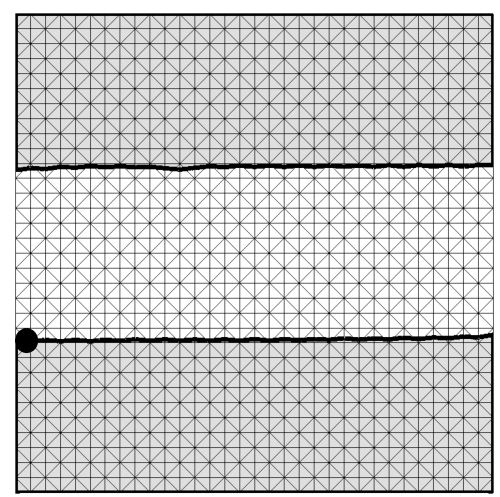

(b) $t=50 \mathrm{~s}$

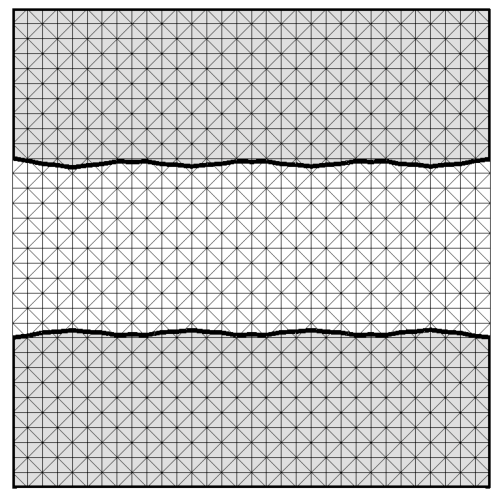

(e) $t=50 \mathrm{~s}$

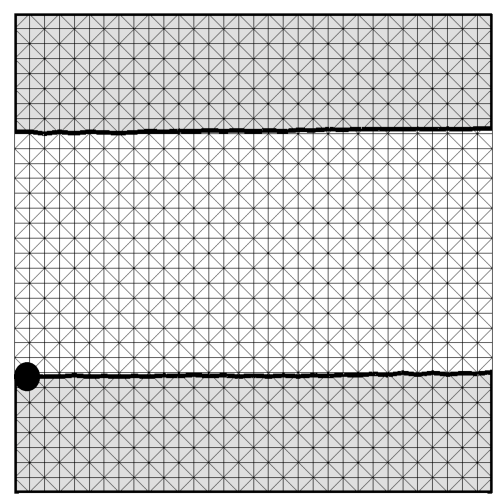

(c) $t=100 \mathrm{~s}$

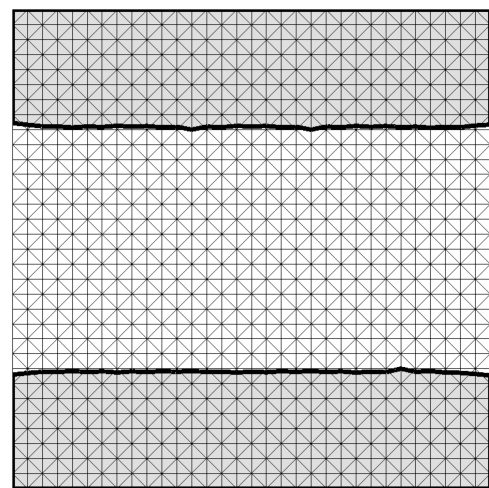

(f) $t=100 \mathrm{~s}$

Fig. 3 Snapshots at different moments of the piping phenomenon, starting from a linear pipe (upper plots) or a pipe with a sine profile (lower plots). The black dots in the upper figures indicate the point that is being followed in the numerical test of figure 6

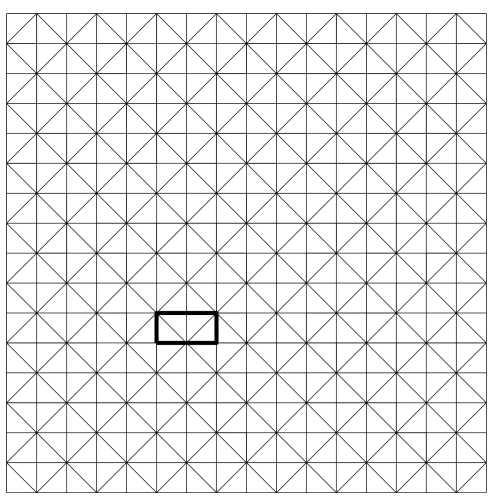

(a) 512 elements, 289 nodes

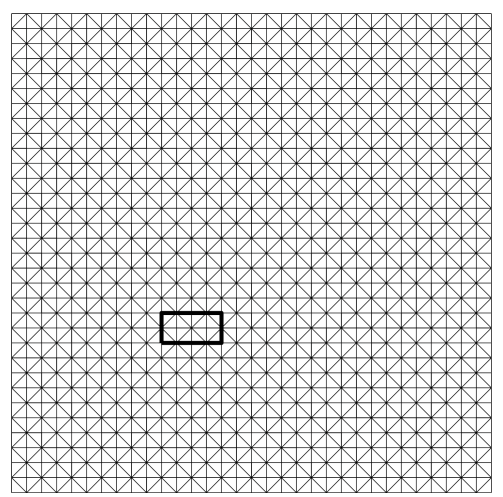

(b) 2048 elements, 1089 nodes

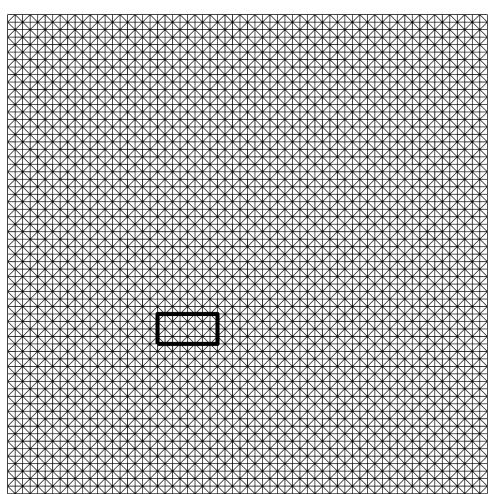

(c) 8192 elements, 4225 nodes

Fig. 4 Three different meshes; the black boxes indicate the zoom displayed in figure 5

We now wish to study the influence of the mesh on the representation of the level-set and on the results obtained. We therefore consider three different meshes, plotted in figure 4, and study, firstly, the influence of the refinement on the shape of the interface, and, secondly, the influence of the refinement on its evolution.

Starting from the sine profile, we therefore study how the interface is described in time, depending on the mesh that is being used. In figure 5, a zoom on that profile is presented at an intermediate time between figures 3(d) and 3(e) ( $t=20 \mathrm{~s}$, when the shape is not flat yet, but the evolution is already visible). On the one hand, it can be observed that, for the cruder mesh, the interface is slightly higher than for the two other meshes. Also, there seems to be some degree of asymmetry. On the other hand, the two finer meshes give representations of the interface that are very similar, and 


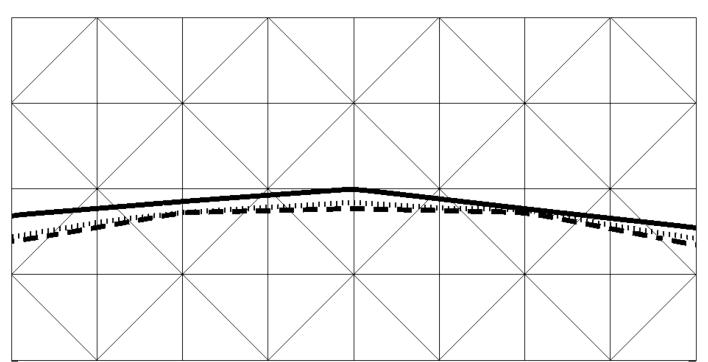

Fig. 5 Influence of the mesh on the shape of the interface at $t=20 \mathrm{~s}$, starting from a sine profile: coarse mesh (solid line, figure 4(a)), fine mesh (dashed line, figure 4(b)), and most refined mesh (dotted line, figure 4(c)). Only the latter mesh is represented (gray lines). This figure is a zoom in the black boxes of figure 4

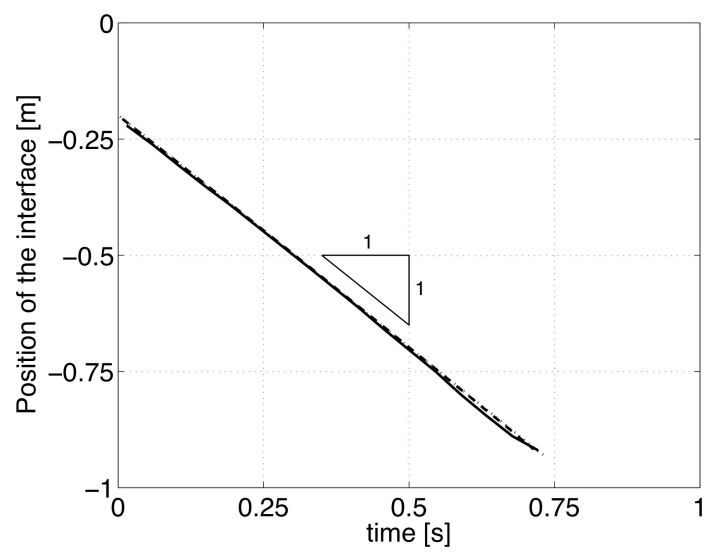

Fig. 6 Influence of the mesh on the resolution of the Hamilton-Jacobi equation: position of the interface (black dot on figure 3 ) as a function of time for a constant velocity $v_{n}=1 \mathrm{~m} / \mathrm{s}$, and for three different meshes: coarse mesh (solid line, figure 4(a)), fine mesh (dashed line, figure 4(b)), and most refined mesh (dotted line, figure 4(c))

symmetric. It therefore seems that the crude mesh is not fine enough, but that both the two other meshes are appropriate for this piping problem.

To go further than these qualitative observations, we then start from the flat profile, and impose a constant velocity in the Hamilton-Jacobi equation. This means that there is no influence of the choice of a given model for the velocity, and only the resolution of the Hamilton-Jacobi is actually scrutinized. Physically, we expect to observe a linear opening of the channel $\Omega_{c}$ with time. Although we do not represent it here, the interface indeed remains flat throughout the computation, and for all meshes. And, in figure 6, we plot the height of the interface (taken at the left-most position in the channel, on the bottom, see the black dot in figure 3) as a function of time. As before, the two finest meshes give results that perfectly overlap each other, and recover the expected velocity of $v_{n}=1 \mathrm{~m} / \mathrm{s}$ (the slope of the curves on figure 6). The coarsest mesh gives results that are almost good but slightly different.

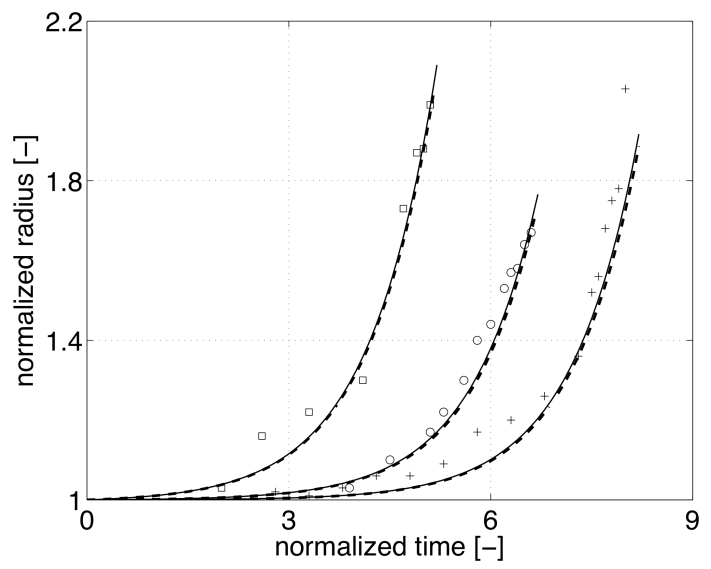

Fig. 7 Value of the normalized radius of a pipe as a function of time computed using the level-set formulation described in this paper (thick dashed lines), using the analytical formula of [43], or using the experimental data from [20] (for tests MDHET006, WBHET001 and HDHET007, respectively in squares, circles and pluses).

Using the more physical model for the velocity of the interface described in [43], again for the case of piping with a flat profile, it is also possible to reproduce very accurately the experimental results obtained in [20] for a wide range of tests. As an example, in figure 7, the analytical value for the radius of the pipe is plotted (in solid line), for three different tests (HDHET007, WBHET001, and MDHET006 in the cited papers) against the experimental values. In dashed lines, the results obtained with our method, and the applied velocity described in [43] reproduces both the analytical and experimental values very accurately. Note that these results were obtained using the intermediate mesh (figure 4(b)). Overall, these results give us confidence in the resolution of the Hamilton-Jacobi equation, and seem to show a good accuracy, even for quite coarse meshes.

\subsection{Modeling of a leaking duct}

We now consider the expansion of a cavity in the earth under the action of a leak in a water duct. For a given level of the water table, it is possible to choose between the two models discussed in sections 2.3 and 2.4. When the water table is low, the cavity if free of water and the leak may be represented as a jet of drops of water. When the water table is higher than the leak, the outgoing water flows in the water retained within the cavity and the earth. However, the outgoing water evacuates to the ground, and eventually affects the level of the water table. In particular, with clay, the evacuation of water is slow and the evolution of the water table level will have to be taken into account. A scheme of the problem is presented in figure 8 , and additional hypotheses are set in the following paragraphs. 


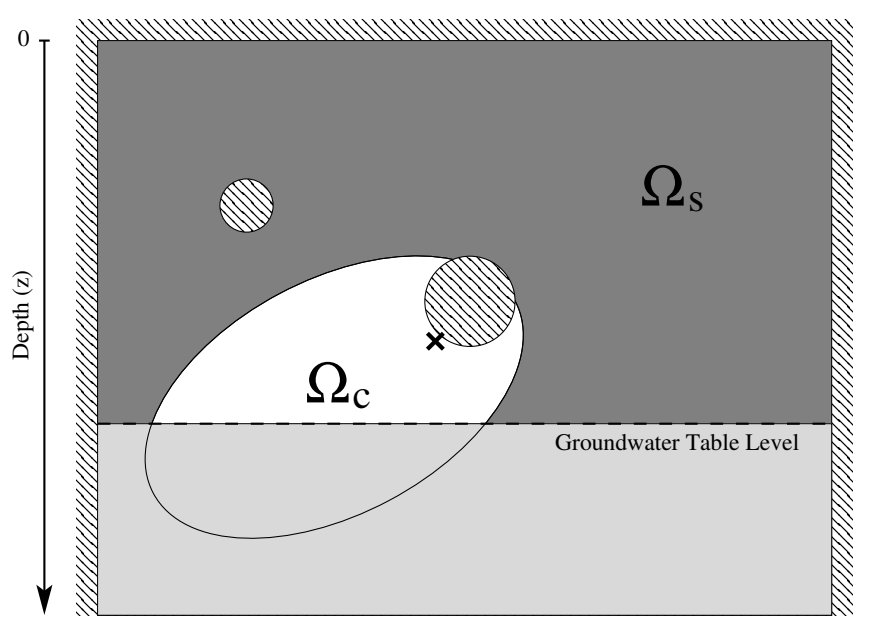

Fig. 8 Modeling of a leaking duct: a cavity $\Omega_{c}$ within a soil $\Omega_{s}$, with no outgoing flux on the left, right and upper sides, two ducts (the dashed holes), one of which leaking (at the location of the star), and a rising water table (dashed line and light grey shade)

Geometry and boundary conditions The problem is modeled in 2 dimensions as a rectangle of dimensions $1.2 \times 1.2$ $\mathrm{m}^{2}$. We suppose that the duct is set below the street level so that the rectangle is bound on the top by a waterproof concrete or asphalt cover. Further, the material in the soil is supposed to be clay, so that the water coming out from the leak cannot evacuate easily. We model this by allowing the water to exit the domain only through the bottom side, and suppose the left and right sides, at some distance from the leak, as waterproof. Finally the duct is taken as waterproof, except in the zone of the leak, where the hydraulic head is set. More precisely, for the flowing phase (high water table), we consider the following boundary conditions

$$
\left\{\begin{array}{ll}
\nabla h(\mathbf{x}, t)=\mathbf{0}, & \mathbf{x} \in \Gamma_{\text {left }} \cup \Gamma_{\text {up }} \cup \Gamma_{\text {right }} \cup\left(\Gamma_{\text {duct }} \backslash \Gamma_{\text {leak }}\right) \\
h(\mathbf{x}, t)=h_{0}, & \mathbf{x} \in \Gamma_{\text {leak }} \\
h(\mathbf{x}, t)=h_{\infty}, & \mathbf{x} \in \Gamma_{\text {bottom }}
\end{array} .\right.
$$

For the impacting phase (low water table), we only consider the erosion phenomenon at impact, and suppose that the water coming out of the jet is evacuated through the bottom part of the domain with no further erosion.

Modeling of the water table We suppose that the level of the water table is the same throughout the domain, that is $z(x, t)=z(t)$. The initial level is taken at some equilibrium level $z\left(t_{0}\right)=z_{\text {ref }}=-1 \mathrm{~m}$. Further, we suppose that the draining substratum can evacuate a flow of $Q_{e}(t)=\alpha S_{0}(z(t)-$ $z_{\text {ref }}$, where $\alpha=10^{-3} \mathrm{~s}^{-1}$ is a material constant, related to the permeability of the soil, and $S_{0}=1.4 \mathrm{~m}^{2}$ is the horizontal surface at the bottom of the model, through which the water is evacuated, supposing a unit length in the hidden dimension. The evolution of the water table then results of a balance between the incoming flow from the leak

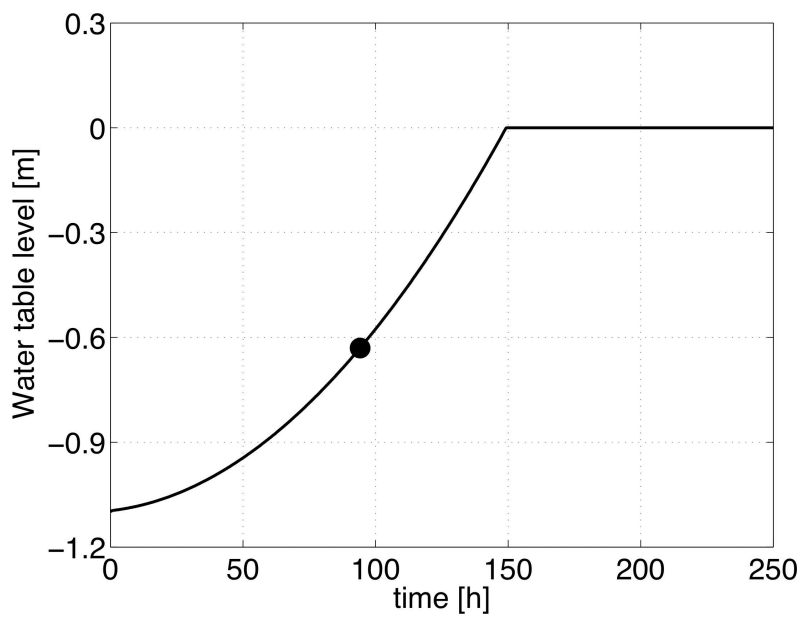

Fig. 9 Evolution of the level of the water table with time. The black dot indicates the change of models for the erosion: jet model for low water table and flow erosion for high water table

$Q_{i}(t)=\pi d_{i}(t)^{2} v_{0} / 4$ and the drained flow $Q_{e}(t)$. The diameter of the hole in the duct is taken as a linear function of time, with $d_{i}(0)=0.4 \mathrm{~mm}$, and $\partial d_{i} / \partial t=1 \mathrm{~mm} /$ day, and $v_{0}=35$ $\mathrm{m} . \mathrm{s}^{-1}$ is a constant that depends only on the pressure of the water in the duct. The balance $S_{0} d z=\left(Q_{i}(t)-Q_{e}(t)\right) d t$ leads to a first order differential equation

$\frac{d z}{d t}+\alpha z=\frac{\pi d_{i}(t)^{2}}{4 S_{0}} v_{0}+\alpha z_{\mathrm{ref}}$

which can be solved, beforehand and explicitly, for any time $t$ (see figure 9, where the level is limited by the waterproof layer at $z=0)$. This model of the water table is therefore solved independently from the problem of the expansion of the cavity and is used only for choosing the appropriate model for the erosion. Note that more complicated models for the opening of the hole in the duct or the draining flow could have been chosen with no additional difficulty.

Low water table - Erosion due to the jet of water While the level of the water table is low enough, the water is projected directly through the air in the cavity to the walls. Following section 2.3, we model the jet of water as a set of drops, and use Eq. (8) for the normal velocity of erosion. Furthermore, we suppose that the drops of water are each coming out from a point $\mathbf{x}_{p 0}=\mathbf{x}_{c}+r_{0} \mathbf{i}_{r}\left(\theta_{p 1}\right)$ at a velocity $\mathbf{v}_{p 0}=v_{0} \mathbf{i}_{r}\left(\theta_{p 2}\right)$. The radius $r_{0}$ of the leaking duct and the outgoing velocity $v_{0}$ are supposed constant for all drops of water. The unit vector $\mathbf{i}_{r}$ is the first basis vector in the classical cylindrical system centered on $\mathbf{x}_{c}=[-0.9-0.7] \mathrm{m}$, the center of the leaking duct, with $\theta_{p 1}$ and $\theta_{p 2}$ uniform random variables with values in $\left[135 \pm d_{i} / 2 r_{0}\right]^{\circ}$ and $[135 \pm 22.5]^{\circ}$, respectively. Finally, in Eq. (8), we take $\mathscr{V}_{0}=10^{-4} \mathrm{~kg}^{-1} \mathrm{~m}^{2} \mathrm{~s}$. 
High water table - Erosion due to a flow When the level of the water table $z(t)$, controlled by Eq. (20), reaches the level of the leak, the jet is damped in the pool of water, and an infiltration process starts. This process is controlled by Darcy's equation (9), with permeabilities defined for the soil and the hollow by Eq. (10) and $K_{s}=7 \times 10^{-8} \mathrm{~cm} \cdot \mathrm{s}^{-1}$. As the interface is moving, the value of the permeability in a given point depends implicitly on time. The normal velocity of the interface is controlled by Eq. (14), with a proportionality coefficient $\lambda=10^{-2} \mathrm{~s}^{-1}$. The boundary conditions for the computation of the hydraulic head gradient are those of Eq. (19). It is reminded that Darcy's law implies a quasistatic hypothesis, which means that the groundwater table is supposed to rise slowly.

Analysis of results Snapshots for the evolution of the cavity in the soil are given in figure 10 at different moments. The initial position of the cavity is given at the first picture. In the first three snapshots, the water table (dashed line) is below the level of the leak so that the erosion happens due to the projection of the drops of water to the far wall of the cavity. Hence the cavity is sculpted to the shape of the jet of water, as indicated by the values taken by the random angles $\theta_{p 1}$ and $\theta_{p 2}$. This shape is particularly well observed in the snapshot (d), taken just before changing to the other model of erosion. Note that the shape is not perfectly symmetrical with respect to the angle $135^{\circ}$, and this is due to the fact that the drops of water undergo the action of gravity. As their velocity is really high, the influence of gravity does not show too much on such a short flight but still skews slightly the shape of the cavity.

When the water table reaches the level of the leak, a pool forms at the bottom of the cavity, which damps the jet of water coming out of the leak. We then shift to the flow model of erosion. The value of the hydraulic head is computed using Darcy's law and the mass conservation equation (9). The boundary conditions used here imply that the flow going out of the leaks tries to evacuate towards the bottom of the model, and hence creates an important flow - and subsequent erosion - from the position of the leak towards the bottom of the model. Therefore the erosion is very small at the furthest part of the cavity (upper left side on the snapshots) and much larger close to the duct. The results of the computation confirm the intuitive analysis.

\section{CONCLUSIONS}

In this paper, a new feature of the level-set representation of the expansion of a hollow in the ground has been described. Namely, the flexibility allowed in terms of modeling was advocated, and backed up by an example where different physical phenomena influence that expansion depending whether the soil is saturated or not. To the knowledge of the authors, this aspect of the level-set representations was not properly illustrated or stressed previously, and constitutes the main novelty of this paper.

Further, the application of level-set methods for the representation of the piping phenomenon was performed. This example involves only one model, and allowed to discuss some aspects related to implementation and numerical accuracy of the level-set representation. Also, it is, to the knowledge of the authors, the first application of level-set methods to the representation of the piping phenomenon, for which it may have a significant impact.

The erosion models used in the examples are rather crude, and aimed at showing the possibilities of the approach presented in the paper. However, it should be emphasized that the level-set representation can be very easily generalized to $3 \mathrm{D}$ problems and even more complex geometries, so that more difficult problems are now within reach.

Acknowledgements The authors would like to acknowledge the help of Dr. S. Zlotnik, who provided the software on which the code developed for this paper was based. Prof. A. Ledesma should also be thanked for giving us a head start in the definition of the flow erosion laws.

\section{References}

1. Crank, J.: Free and moving boundary problems. Oxford University Press (1984)

2. Unverdi, S.O., Tryggvason, G.: A front-tracking method for viscous, incompressible, multi-fluid flows. Journal of Computational Physics 100(1), 25-37 (1992). DOI 10.1016/00219991(92)90307-K

3. Gorczyk, W., Gerya, T.V., Connolly, J.A., Yuen, D.A., Rudolph, M.: Large-scale rigid-body rotation in the mantle wedge and its implications for seismic tomography. Geochemistry Geophysics Geosystems 7(5), Q05,018 (2006). DOI 10.1029/2005GC001075

4. Jan, Y.J.: A cell-by-cell thermally driven mushy cell tracking algorithm for phase-change problems. Computational Mechanics 40(2), 201-216 (2007). DOI 10.1007/s00466-006-0098-x

5. van Keken, P.E., King, S.D., Schmeling, H., Christensen, U.R., Neumeister, D., Doin, M.P.: A comparison of methods for the modeling of thermochemical convection. Physics of Fluids A 102(B10), 22,477-22,496 (1997). DOI 10.1029/97JB01353

6. Sethian, J.A.: Level set methods and fast marching methods. Cambridge University Press (1999)

7. Osher, S., Fedkiw, R.P.: Level set methods: an overview and some recent results. Journal of Computational Physics 169(2), 463-502 (2001). DOI 10.1006/jcph.2000.6636

8. Osher, S., Sethian, J.A.: Fronts propagating with curvaturedependent speed: Algorithms based on Hamilton-Jacobi formulations. Journal of Computational Physics 79(1), 12-49 (1988). DOI 10.1016/0021-9991(88)90002-2

9. Sussman, M., Smereka, P., Osher, S.: A level set approach for computing solutions to incompressible two-phase flow. Journal of Computational Physics 114(1), 146-159 (1994). DOI 10.1006/jcph.1994.1155

10. Mulder, W., Osher, S., Sethian, J.A.: Computing interface motion in compressible gas dynamics. Journal of Computational Physics 100(2), 209-228 (1992). DOI 10.1016/0021-9991(92)90229-R

11. Karlsen, K.H., Lie, K.A., Risebro, N.H.: A fast marching method for reservoir simulation. Computational Geosciences 4(2), 185206 (2000). DOI 10.1023/A:1011564017218 


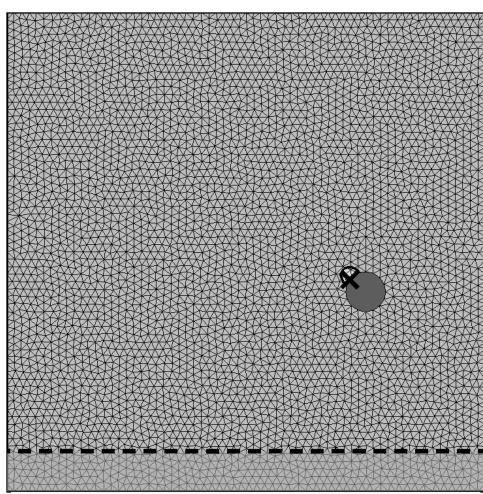

(a) $t=0 \mathrm{~h}$

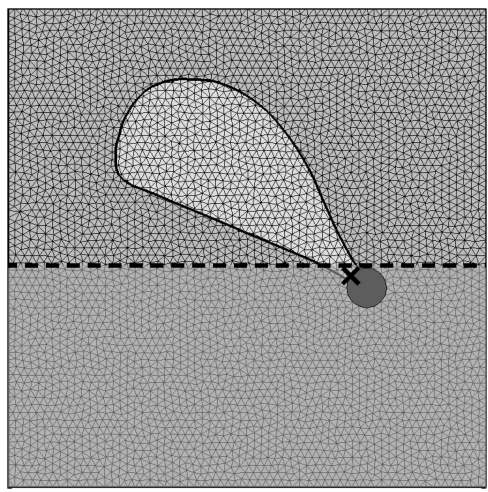

(d) $t=90 \mathrm{~h}$

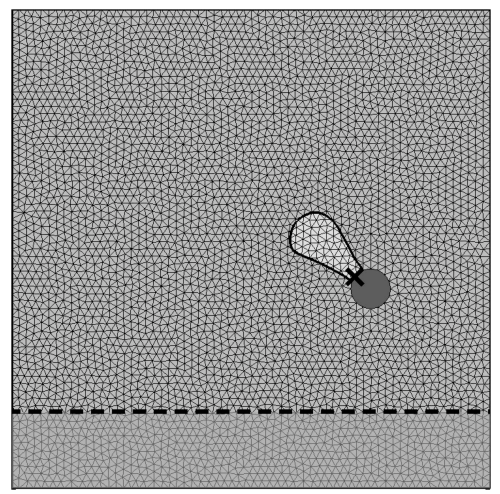

(b) $t=30 \mathrm{~h}$

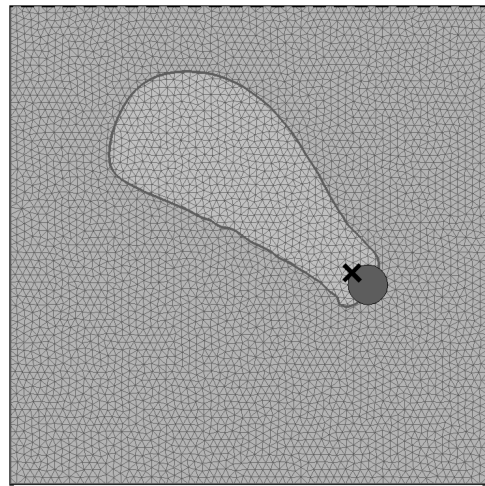

(e) $t=150 \mathrm{~h}$

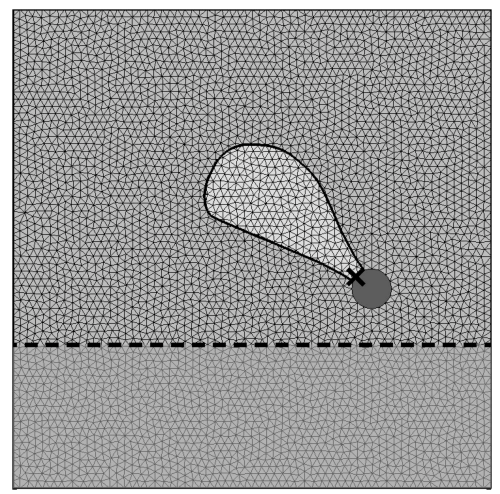

(c) $t=60 \mathrm{~h}$

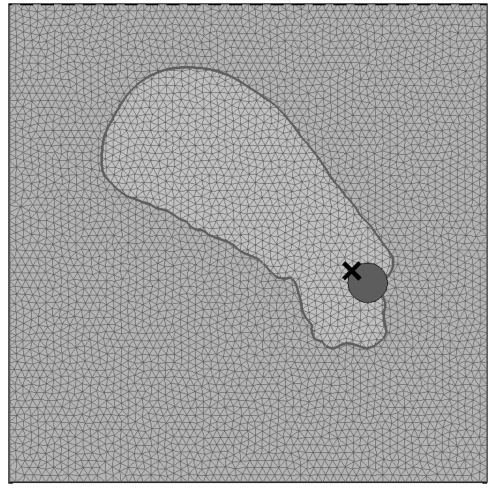

(f) $t=225 \mathrm{~h}$

Fig. 10 Snapshots at different moments of the hollow (light grey shade) in the ground (dark grey shade) created by a leaking duct (the duct is the dark grey circle and the leak is the black cross). Before (d), the water table (dashed line) is below the hole in the duct and the jet model is used. After (d), the flow model is used.

12. Nielsen, L.K., Li, H., Tai, X.C., Aanonsen, S.I., Espedal, M.: Reservoir description using a binary level set model. Computing and Visualization in Science (2008). DOI 10.1007/s00791-0080121-1

13. Zlotnik, S., Díez, P., Fernández, M., Vergés, J.: Numerical modelling of tectonic plates subduction using X-FEM. Computer Methods in Applied Mechanics and Engineering 196(41-44), 4283-4293 (2007). DOI 10.1016/j.cma.2007.04.006

14. Zlotnik, S., Fernández, M., Díez, P., Vergés, J.: Modelling gravitational instabilities: slab break-off and Rayleigh-Taylor diapirism. Pure and Applied Geophysics 165, 1-20 (2008). DOI 10.1007/s00024-004-0386-9

15. Sethian, J.A., Popovici, A.M.: 3-D traveltime computation using the fast marching method. Geophysics 64(2), 516-523 (1999). DOI 10.1190/1.1444558

16. Ito, K., Kunisch, K., Li, Z.: Level-set function approach to an inverse interface problem. Inverse Problems 17(5), 1225-1242 (2001). DOI 10.1088/0266-5611/17/5/301

17. Burger, M.: A level set method for inverse problems. Inverse Problems 17(5), 1327-1355 (2001). DOI 10.1088/0266-5611/17/5/307

18. Burger, M., Osher, S.J.: A survey of level set methods for inverse problems and optimal design. European Journal of Applied Mathematics 16(2), 263-301 (2005). DOI 10.1017/S0956792505006182

19. Bonelli, S., Brivois, O., Borghi, R., Benahmed, N.: On the modelling of piping erosion. Comptes-Rendus Mécanique 334(8-9), 555-559 (2006). DOI 10.1016/j.crme.2006.07.003

20. Wan, C.F., Fell, R.: Investigation of rate of erosion of soils in embankment dams. Journal of Geotechnical and Geoenvi- ronmental Engineering, ASCE 130(4), 373-380 (2004). DOI 10.1061/(ASCE)1090-0241(2004)130:4(373)

21. Wan, C.F., Fell, R.: Laboratory tests on the rate of piping erosion of soils in embankment dams. Geotechnical Testing Journal 27(3), 1-9 (2004). DOI 10.1520/GTJ11903

22. Zlotnik, S., Díez, P.: Hierarchical X-FEM for n-phase flow ( $\mathrm{n}>2$ ). Computer Methods in Applied Mechanics and Engineering 198(30-32), 2329-2338 (2009). DOI 10.1016/j.cma.2009.02.025

23. Darcy, H.: Les Fontaines Publiques de la Ville de Dijon. Dalmon, Paris (1856)

24. Bear, J., Bachmat, Y.: Introduction to mmodeling of transport phenomena in porous media. In: J. Bear (ed.) Theory and Applications of Transport in Porous Media, vol. 4, p. 136. Kluwer Academic Publishers (1999)

25. Knapen, A., J.Poesen, Govers, G., Gyssels, G., Nachtergaele, J.: Resistance of soils to concentrated flow erosion: a review. Earth-Science Reviews 80(1-2), 75-109 (2007). DOI 10.1016/j.earscirev.2006.08.001

26. Vardoulakis, I., Stavropoulou, M., Papanastasiou, P.: Hydromechanical aspects of the sand production problem. Transport in Porous Media 22(2), 225-244 (1996). DOI 10.1007/BF01143517

27. Vardoulakis, I., Papanastasiou, P., Stavropoulou, M.: Sand erosion in axial flow conditions. Transport in Porous Media 45(2), 267280 (2001). DOI 10.1023/A:1012035031463

28. Giménez, R., Govers, G.: Flow detachment by concentrated flow on smooth and irregular beds. Soil Science Society of America Journal 66, 1475-1483 (2002)

29. Woodward, D.E.: Method to predict cropland ephemeral gully erosion. CATENA 37(3-4), 393-399 (1999). DOI 10.1016/S03418162(99)00028-4 
30. Sidorchuk, A.: Stochastic components in the gully erosion modelling. CATENA 63(2-3), 299-317 (2005). DOI 10.1016/j.catena.2005.06.007

31. Donea, J., Huerta, A.: Finite Element Methods for Flow Problems. John Wiley \& sons Ltd., Chichester (2003)

32. Adalsteinsson, D., Sethian, J.A.: The fast construction of extension velocities in level set methods. Journal of Computational Physics 148(1), 2-22 (1999). DOI 10.1006/jcph.1998.6090

33. Russo, G., Smereka, P.: A remark on computing distance functions. Journal of Computational Physics 163(1), 51-67 (2000). DOI $10.1006 /$ jcph. 2000.6553

34. Rouy, E., Touring, A.: A viscosity solutions approach to shapefrom-shading. SIAM Journal on Numerical Analysis 29(3), 867884 (1992). DOI 10.1137/0729053

35. Budyn, E., Hoc, T., Jonvaux, J.: Fracture strength assessment and aging signs detection in human cortical bone using an X-FEM multiple scale approach. Computational Mechanics 42(4), 579591 (2008). DOI 10.1007/s00466-008-0283-1

36. Yvonnet, J., Quang, H.L., He, Q.C.: An XFEM/level set approach to modelling surface/interface effects and to computing the sizedependent effective properties of nanocomposites. Computational Mechanics 42(1), 119-131 (2008). DOI 10.1007/s00466-0080241-y

37. Gross, S., Reusken, A.: Finite element discretization error analysis of a surface tension force in two-phase incompressible flows. SIAM Journal on Numerical Analysis 45(4), 1679-1700 (2007). DOI $10.1137 / 060667530$

38. Legrain, G., Moës, N., Huerta, A.: Stability of incompressible formulations enriched with X-FEM. Computer Methods in Applied Mechanics and Engineering 197(21-24), 1835-1849 (2008). DOI 10.1016/j.cma.2007.08.032

39. Bligh, W.G.: Dams, barrages and weirs on porous foundations. Engineering News 64, 708 (1910)

40. Lane, E.W.: Security from under-seepage masonry dams on earth foundations. Transaction of the ASCE 100, 1235-1351 (1935)

41. Koenders, M.A., Sellmeijer, J.B.: A mathematical model for piping. Journal of Geotechnical Engineering 118(6), 943-946 (1992). DOI 10.1061/(ASCE)0733-9410(1992)118:6(943)

42. Ojha, C.S., Singh, V.P., Adrian, D.D.: Determination of critical head in soil piping. Journal of Hydraulic Engineering, ASCE 129(7), 511-518 (2003). DOI 10.1061/(ASCE)07339429(2003)129:7(511)

43. Bonelli, S., Brivois, O.: The scaling law in the hole erosion test with a constant pressure drop. International Journal for Numerical and Analytical Methods in Geomechanics 32(13), 1573-1595 (2008). DOI 10.1002/nag.683

44. Lachouette, D., Golay, F., Bonelli, S.: One-dimensional modeling of piping flow erosion. Comptes-Rendus Mécanique 336(9), 731736 (2008). DOI 10.1016/j.crme.2008.06.007

45. Burns, B., Barker, R., Ghataora, G.S.: Investigating internal erosion using a miniature resistivity array. NDT \& E International 39(2), 169-174 (2006). DOI 10.1016/j.ndteint.2004.12.009 